\title{
Iron-biofortification in rice by the introduction of three barley genes participated in mugineic acid biosynthesis with soybean ferritin gene
}

\author{
Hiroshi Masuda ${ }^{1,2}$, Takanori Kobayashi ${ }^{1,2}$, Yasuhiro Ishimaru ${ }^{2}$, Michiko Takahashi $^{2}$, May S. Aung $^{2}$, \\ Hiromi Nakanishi ${ }^{2}$, Satoshi Mori ${ }^{2}$ and Naoko K. Nishizawa ${ }^{1,2 *}$
}

${ }^{1}$ Research Institute for Bioresources and Biotechnology, Ishikawa Prefectural University, Ishikawa, Japan

${ }^{2}$ Graduate School of Agricultural and Life Sciences, The University of Tokyo, Tokyo, Japan

\section{Edited by:}

Michael A. Grusak, USDA-ARS

Children's Nutrition Research

Center, USA

Reviewed by:

Stanislav Kopriva, John Innes

Centre, UK

Rubén Rellán-Álvarez, Carnegie Institution for Science, USA

*Correspondence:

Naoko K. Nishizawa, Research Institute for Bioresources and

Biotechnology, Ishikawa Prefectural

University, 1-308 Suematsu,

Nonoichi, Ishikawa, 921-8836,

Japan.

e-mail:annaoko@mail.

ecc.u-tokyo.ac.jp
Iron deficiency is a serious problem around the world, especially in developing countries. The production of iron-biofortified rice will help ameliorate this problem. Previously, expression of the iron storage protein, ferritin, in rice using an endosperm-specific promoter resulted in a two-fold increase in iron concentration in the resultant transgenic seeds. However, further over expression of ferritin did not produce an additional increase in the seed iron concentration, and symptoms of iron deficiency were noted in the leaves of the transgenic plants. In the present study, we aimed to further increase the iron concentration in rice seeds without increasing the sensitivity to iron deficiency by enhancing the uptake and transport of iron via a ferric iron chelator, mugineic acid. To this end, we introduced the soybean ferritin gene (SoyferH2) driven by two endosperm-specific promoters, along with the barley nicotianamine synthase gene (HVNAS1), two nicotianamine aminotransferase genes (HVNAAT-A and -B), and a mugineic acid synthase gene (IDS3) to enhance mugineic acid production in rice plants. A marker-free vector was utilized as a means of increasing public acceptance. Representative lines were selected from 102 transformants based on the iron concentration in polished seeds and ferritin accumulation in the seeds. These lines were grown in both commercially supplied soil (iron-sufficient conditions) and calcareous soil (iron-deficient conditions). Lines expressing both ferritin and mugineic acid biosynthetic genes showed signs of iron-deficiency tolerance in calcareous soil. The iron concentration in polished $T_{3}$ seeds was increased by 4 and 2.5 times, as compared to that in non-transgenic lines grown in normal and calcareous soil, respectively. These results indicate that the concomitant introduction of the ferritin gene and mugineic acid biosynthetic genes effectively increased the seed iron level without causing iron sensitivity under iron-limited conditions.

Keywords: anemia, iron, zinc, rice, mugineic acid, biofortification, ferritin, IDS3

\section{INTRODUCTION}

Iron $(\mathrm{Fe})$ is an essential micronutrient for most organisms, including all plants and animals. Fe deficiency is one of the most prevalent micronutrient deficiencies in the world, affecting an estimated two billion people (Stoltzfus and Dreyfuss, 1998) and causing 0.8 million deaths per year worldwide. Fe deficiency is ranked sixth among the risk factors for death and disability in developing countries (WHO, 2002).

There are three basic ways to solve micronutrient deficiencies: micronutrient supplementation, food fortification, and biofortification. Among these options, biofortification does not require specific processing after harvest or a special infrastructure (Grusak and DellaPenna, 1999; Mayer et al., 2008). Therefore, it is beneficial for people who may find it difficult to change their dietary habits because of financial, cultural, regional, or religious restrictions. Rice is a particularly suitable target for biofortification because Fe-deficiency anemia is a serious problem in developing countries where rice is a major staple crop (Juliano, 1993; WHO, 2002). Rice endosperm accumulates a high concentration of starch and becomes the edible part of the seed after milling, at which point the seeds are known as polished or white seeds (Juliano, 1993). Rice seeds, and especially endosperm, contain low levels of most minerals, including micronutrient metals (Grusak and Cakmak, 2005); therefore, it is important to improve the $\mathrm{Fe}$ concentration in polished seeds.

There are many trials to improve mineral nutrition in rice seeds by traditional breeding or transgenic methods. IR68144 was produced using traditional breeding by International Rice Research Institute (IRRI). This variety contains over two times higher Fe concentration in seeds than local varieties in Philippine (Gregorio et al., 2000). IR68144 improved the Fe states of 
Philippine women better than a local rice variety (Haas et al., 2005).

Nowadays, transgenic approach can be used for the production of micronutrient-fortified rice varieties. The first transgenic approach to increase Fe concentration in rice seeds is the enhancement of $\mathrm{Fe}$ accumulation in rice seeds by ferritin gene expression under the control of endosperm-specific promoters. Goto et al. (1999) generated transgenic rice plants that expressed the soybean ferritin gene, SoyferH1, in endosperm using the endosperm-specific, 1.3-kb OsGluB1 rice promoter; the transformants showed increased $\mathrm{Fe}$ accumulation in brown seeds. A few reports have described the production of Fe-biofortified rice through the endosperm-specific expression of ferritin (Lucca et al., 2002; Vasconcelos et al., 2003). Furthermore, Qu et al. (2005) expressed SoyferH1 under the control of both the OsGlb promoter and 1.3-kb OsGluB1 promoter to further increase the seed Fe concentration. However, increasing the level of ferritin expression in rice seeds did not significantly increase the Fe concentration; moreover, it caused symptoms of iron deficiency in the leaves of the transgenic plants. Thus, the enhancement of ferritin expression may not be sufficient to further increase the Fe concentration in rice grains. Qu et al. (2005) proposed that in addition to increased Fe storage in seeds, enhanced Fe uptake from the soil and enhanced translocation within the plant body are required to further improve the Fe biofortification of rice seeds.

Fe uptake, translocation, and homeostasis in rice are beginning to be understood at the molecular level (Grusak et al., 1999; Bashir et al., 2010). Graminaceous plants synthesize and secrete mugineic acid family phytosiderophores (MAs), which are natural $\mathrm{Fe}(\mathrm{III})$ chelators that take up Fe from the rhizosphere (Figure S1; Takagi, 1976; Mihashi and Mori, 1989). Nicotianamine (NA) is biosynthesized from $S$-adenosyl methionine via NAS (Higuchi et al., 1999). In graminaceous plants, including rice, deoxymugineic acid (DMA) is synthesized from NA by NA aminotransferase (NAAT) and DMA synthase (DMAS) (Takahashi et al., 1999; Bashir et al., 2006; Inoue et al., 2009). In barley and other graminaceous plants, other types of MAs are synthesized from DMA by Fe deficiency-specific clone no. 2 (IDS2) and no. 3 (IDS3: also known as mugineic acid synthase) (Nakanishi et al., 2000; Kobayashi et al., 2001). Among graminaceous plants, barley is highly tolerant to Fe deficiency and possesses a series of biosynthetic genes for MAs, including HvNAS1, $H v N A A T-A, H v N A A T-B, H v D M A S 1, I D S 2$, and IDS3, which are up-regulated in Fe-deficient barley roots (Higuchi et al., 1999; Takahashi et al., 1999; Nakanishi et al., 2000; Bashir et al., 2006). In contrast, rice lacks IDS2 and IDS3 and secretes only DMA. This is thought to be one of the reasons why barley has greater tolerance to Fe deficiency than rice (Kobayashi et al., 2001). In rice, $\mathrm{Fe}$ (III)-DMA complexes are thought to be absorbed through the transporter OsYSL15 (Inoue et al., 2009; Lee et al., 2009a). In addition to its function in Fe uptake, Fe(III)-DMA is transported into rice seeds more efficiently, as compared to $\mathrm{Fe}$ (III) through the rice plant body (Tsukamoto et al., 2009). Based on our knowledge of the mechanism of Fe uptake and transport by MAs in graminaceous plants, transgenic rice lines with increased tolerance to Fe deficiency were produced. Suzuki et al. (2008) cultivated three types of transgenic rice lines carrying the barley genes responsible for MAs biosynthesis ( $H v N A S 1, H v N A S 1$, $H v N A A T-A, H v N A A T-B$, and IDS3) in a field with calcareous soil. Rice lines expressing HvNAS1 or IDS3 showed Fe-deficiency tolerance, possibly because of improved Fe uptake and translocation caused by the enhancement of DMA and MA biosynthesis. In addition to DMA, the introduction of IDS3 conferred MA secretion in rice (Kobayashi et al., 2001). Because MA have greater $\mathrm{Fe}(\mathrm{III})$-complex stability than DMA at a slightly acidic $\mathrm{pH}$ (von Wirén et al., 2000), the production of MA via IDS3 might be advantageous for Fe translocation in rice. Furthermore, because these transformants contained introduced barley genome fragments, expression of the genes responsible for MAs biosynthesis was regulated by their own promoters. In rice, these promoters induced expression in response to Fe deficiency in roots and leaves (Higuchi et al., 2001; Kobayashi et al., 2001). Thus, these genes are expected to be expressed when and where the requirement for $\mathrm{Fe}$ is elevated.

The Fe concentration in seeds of rice lines transformed with $H v N A S 1, H v N A S 1, H v N A A T-A$, HvNAAT-B, and IDS3 was analyzed after cultivation in the field in Fe-sufficient (Andosol) or Fe-deficient (calcareous) soil (Masuda et al., 2008; Suzuki et al., 2008). The IDS3 rice line showed an increased Fe concentration in polished seeds up to 1.25-1.4 times that in non-transgenic (NT) rice following cultivation in Andosol and calcareous soil (Masuda et al., 2008; Suzuki et al., 2008).

In the present report, we produced Fe biofortified rice by the concomitant introduction of soybean ferritin gene (SoyferH2) under the control of the OsGluB1 and OsGlb promoters and barley genes encoding enzymes for MAs biosynthesis (genome fragments of $H v N A S 1, H v N A A T-A, H v N A A T-B$, and IDS3). The transformants exhibited Fe-deficiency tolerance in calcareous soil. The $\mathrm{Fe}$ concentration in $\mathrm{T}_{3}$ polished seeds was increased 4 and 2.5 times, as compared to that in NT plants grown in commercially supplied soil and calcareous soil, respectively. We found that Fe biofortification through the concomitant introduction of genes encoding ferritin and biosynthetic enzymes for MAs effectively increased the seed Fe level and improved Fe sensitivity under Fe limitation, which is caused in case of single introduction of ferritin.

\section{MATERIALS AND METHODS PLANT MATERIALS}

The Japonica rice (Oryza sativa L.) cultivar Tsukinohikari was used as the NT control and for transformation.

\section{VECTOR CONSTRUCTION, CONFIRMATION OF VECTOR CONSTRUCT AND RICE TRANSFORMATION}

pBIMFN (marker-free vector), which was produced by Nishizawa et al. (2006), was used as the backbone of the binary vector for rice transformation. Using this vector, the Fer-NAS-NAAT-IDS3 and Fer rice transformation vectors were constructed according to the scheme shown in Figures S2, S3, respectively. The constructed vectors were verified by PCR, as shown in Figure S4. For Fer-NAS-NAAT-IDS3 vector, Glbp 5'R primer 5'-ACC AGA TAC AAC GGG TCC CTC- $3^{\prime}$ and NAAT $5^{\prime} \mathrm{R}$ primer $5^{\prime}-\mathrm{GGT}$ ATC GCC ATT CGC CAA GCC AGT-3' were used to confirm 
the gene connection of gene cassette OsGlb promoter-SoyferH2 and $H v N A A T-A,-B$. NAAT $3^{\prime} \mathrm{F}$ primer $5^{\prime}$-GTC ACT CGC TCT ATC TTG GTC ATT G-3' and NAS 5'R primer 5'-GTT GAG GAT ACA CTA TTG CTC ATG C-3' were used to confirm the gene connection of $H v N A A T-A,-B$ genome and $H v N A S 1$ genome. NAS $3^{\prime} \mathrm{F}$ primer $5^{\prime}$-GAC TAA GCG TCG TCA TGA ACC TGT G-3' and tNos $3^{\prime} \mathrm{F}$ primer $5^{\prime}$-GAA TCC TGT TGC CGG TCT TGC G-3' were used to confirm the gene connection of HvNAS1 genome and OsGluB1 promoter-SoyferH2 gene construct. GluBp 5'R primer 5'-TGA ACA GTC GTG CTC ACG GTC-3' and IDS3g $5^{\prime} \mathrm{R}$ primer $5^{\prime}$-AAC ACA GTA TAG ACG CAA GTG TTC A- $3^{\prime}$ were used to confirm the gene connection of OsGluB1 promoter-SoyferH2 gene construct and IDS3 genome. For Fer vector, Glbp $5^{\prime} \mathrm{R}$ primer and GluBp $5^{\prime} \mathrm{R}$ primer were used to confirm the gene connection of gene cassette OsGlb promoter-SoyferH2 and OsGluB1 promoter-SoyferH2. Sequence of PCR product was checked by ABI PRISM 310(ABI) and compared to the expected sequence from the data.

Agrobacterium tumefaciens (strain C58) was used to introduce the constructs into O. sativa $\mathrm{L}$. cv. Tsukinohikari using the method outlined in Hiei et al. (1994). Transgenic calli were serially selected by 10,20 , and $30 \mathrm{mg} / \mathrm{L}$ concentrations of hygromycin. $30 \mathrm{mg} / \mathrm{L}$ concentration of hygromycin was also included in regeneration medium and root elongation medium.

\section{GREENHOUSE CULTIVATION}

$\mathrm{T}_{0}$ regenerate plants as well as $\mathrm{T}_{1}$ Fer-NAS-NAAT-IDS3 lines, Fer lines, and NT plants were germinated on Murashige and Skoog (MS) medium and cultivated in 3.5 CL pots (1,000-ml volume; Kaneya, Aichi, Japan) containing a 2:1 mixture of Bonsol-ichigou (commercially supplied soil used for rice cultivation in Japanese nurseries; Sumitomo Chemicals, Tokyo, Japan) and vermiculite (Green Tec, Tochigi, Japan). The soil was evenly fertilized with $3.5 \mathrm{~g}$ of Long Total-70 and Long Total-140 (slow-release fertilizers; JCAM AGRI Co. Ltd., Tokyo, Japan; N:P:K, 13:11:13 and $2 \% \mathrm{Mg}, 0.1 \% \mathrm{Mn}, 0.06 \% \mathrm{~B}, 0.20 \% \mathrm{Fe}, 0.050 \% \mathrm{Cu}, 0.015 \% \mathrm{Zn}$, and $0.020 \%$ Mo as micronutrients) per plant. The plants were grown in a greenhouse under natural light conditions, with $14 \mathrm{~h}$ of light at $30^{\circ} \mathrm{C}$ and $10 \mathrm{~h}$ of dark at $25^{\circ} \mathrm{C}$. Six plants each of the $\mathrm{T}_{2}$ Fer-NAS-NAAT-IDS3 lines (1-12, 22-4, and 34-11), Fer line 13-6, and NT plants were cultivated in commercially supplied soil and vermiculite as described above under Fe-sufficient conditions. The $\mathrm{T}_{2}$ plants were also cultivated in calcareous soil $(\mathrm{pH}=8.9$ ) obtained from Takaoka City, Toyama, Japan (Nihonkai Kougyo, Toyama, Japan) in $3.5 \mathrm{CL}$ pots with $3.5 \mathrm{~g}$ of Long Total-70 and -140 under Fe-deficient conditions. The seeds obtained from greenhouse cultivation were used for metal concentration analysis.

For Northern blot analysis, $\mathrm{T}_{2}$ Fer-NAS-NAAT-IDS3 lines 112, 22-4, and 34-11 were germinated on MS medium. After 3 weeks, six seedlings from each line were transferred to nutrient solution $\left[2 \mathrm{mM} \mathrm{Ca}\left(\mathrm{NO}_{3}\right)_{2}, 0.5 \mathrm{mM} \mathrm{MgSO}_{4}, 0.7 \mathrm{mM} \mathrm{K}_{2} \mathrm{SO}_{4}\right.$, $0.1 \mathrm{mM} \mathrm{KCl}, 0.1 \mathrm{mM} \mathrm{KH} 2 \mathrm{PO}_{4}, 0.1 \mathrm{mM}$ Fe(III)-EDTA, $10 \mathrm{mM}$ $\mathrm{H}_{3} \mathrm{BO}_{3}, 0.5 \mu \mathrm{M} \mathrm{MnSO}, 0.5 \mu \mathrm{M} \mathrm{ZnSO}_{4}, 0.2 \mu \mathrm{M} \mathrm{CuSO}$, and $\left.0.01 \mu \mathrm{M}\left(\mathrm{NH}_{4}\right)_{6} \mathrm{Mo}_{7} \mathrm{O}_{25}\right]$ and grown in a greenhouse under the conditions described above. The $\mathrm{pH}$ of the culture solution was adjusted daily to between 5.5 and 5.8 with $1 \mathrm{~N} \mathrm{HCl}$. After 8 days, the plants were transplanted to fresh nutrient solution without Fe(III)-EDTA and cultivated for 1 week. Next, the leaf chlorophyll level was measured using a SPAD-502 chlorophyll meter (Konica Minolta, Tokyo, Japan), and leaves and roots were harvested for Northern blot analysis.

\section{GENOMIC PCR}

For each line, leaf samples were harvested and DNA was extracted using an automated genomic DNA isolation system (NA-2000; Kurabo, Osaka, Japan). Next, genomic PCR was performed using the following primers. The OsGluB1 promoter SoyferH2 construct was detected using the OsGluB1 promoter FW primer $\left(5^{\prime}\right.$-CAG CTC TCC GTG GTC AGA TGT G-3') and SoyferH2 RV primer (5'-GCC ACA CAC CAT GAC CCT TTC CAA C- $\left.3^{\prime}\right)$. The OsGlb promoter SoyferH2 construct was detected using the OsGlb promoter FW primer (5'-CCA ACC GAT CCA TGT CAC CCT CAA GC-3') and SoyferH2 RV primer. IDS3 genome insertion was detected using the IDS3 FW primer (5'-AAG CTT ACT GGT TGG ACG GTA TTT CA-3') and IDS3 RV primer (5'-GGA TCC ACG GGC CAC ATG ATC CA-3'). HvNAAT-A genome insertion was detected using the $H v N A A T-A$ FW primer $\left(5^{\prime}-\mathrm{GTG}\right.$ TTG CAT GTC AAA TGA CCG G-3') and $H v N A A T-A$ RV primer $\left(5^{\prime}-\right.$ CTA CTC CCT CTG TCC CAA AAT AAC TG-3'). HvNAAT-B genome insertion was detected using the $H v N A A T-B$ FW primer ( $5^{\prime}$-CCG AAA ATG CAT CCA ACA TAA TTA C- $3^{\prime}$ ) and $H v N A A T$ $B$ RV primer (5'-GCC AAT GTA ACT TCA CTA ACA TAA C-3'). $H v N A S 1$ genome insertion was detected using the HvNAS1 FW primer (5'-CGG TGG AGG TAA TAG CCC TAC GTC-3') and HvNAS1 RV primer (5'- GGA GGC AGT CCT GTT GTG GCA TTC-3').

\section{NORTHERN BLOT ANALYSIS}

The ORFs for HvNAS1 (AB010086), HvNAAT-A (D88273), $H v N A A T-B$ (AB005788), and IDS3 (AB024058) were used to synthesize $H v N A S 1, H v N A A T-A, H v N A A T-B$, and IDS3 probes using the primers described in Suzuki et al. (2006). This fragment was labeled with $\left[\mathrm{a}^{32} \mathrm{P}\right]$-dATP using the random labeling method; the labeled DNA was purified using a ProbeQuant G-50 Micro Column (Pharmacia, Uppsala, Sweden). Total RNA from the roots and shoots was extracted using the sodium dodecyl sulfate (SDS)-phenol method. Aliquots of the total RNA (20 $\mu \mathrm{g}$ per lane) were separated on $1.4 \%(\mathrm{w} / \mathrm{v})$ agarose gels. Blotting, hybridization, and radioactive detection were performed as described previously (Ogo et al., 2006; Suzuki et al., 2006).

\section{WESTERN BLOT ANALYSIS}

Six $\mathrm{T}_{2}$ or six $\mathrm{T}_{3}$ mature seeds of the Fer-NAS-NAAT-IDS3, Fer, and NT lines were harvested and examined for ferritin expression by Western blotting. The seeds were homogenized with a mortar and pestle, soaked in extraction buffer (4\% SDS, 5\% 2-mercaptethanol, 20\% glycerol, $20 \mathrm{mM}$ Tris- $\mathrm{HCl}, 8 \mathrm{M}$ urea, and $0.1 \%$ bromophenol blue, $\mathrm{pH} 6.8$ ), and shaken for $30 \mathrm{~min}$. The resulting extracts were centrifuged at $13,000 \mathrm{rpm}$ for $20 \mathrm{~min}$ and supernatant fractions were collected. Protein separation by SDSpolyacrylamide gel electrophoresis, transfer to polyvinylidene fluoride membranes, and detection with antibodies were performed as described in Goto et al. (1999). 


\section{QUANTITATIVE REAL-TIME RT-PCR ANALYSIS OF SoyferH2 IN FE DEFICIENT LEAVES}

Plants were germinated on MS medium. After 3 weeks, three plants from each line were cultivated in nutrient solution with $\mathrm{Fe}(\mathrm{III})$-EDTA for 2 weeks and then in nutrient solution without Fe(III)-EDTA for 13 days. Total RNA was extracted from the second newest leaf of each plant by using an RNeasy Plant Mini Kit (Qiagen, Tokyo, Japan). First-strand cDNA was synthesized using ReverTra Ace qPCR RT Master Mix with gDNA remover (TOYOBO, Osaka, Japan). Real-time RT-PCR was carried out using the StepOnePlus ${ }^{\mathrm{TM}}$ Real-Time PCR System (Applied Biosystems, Tokyo, Japan) with SYBR Premix Ex Taq II (Takara, Shiga, Japan). SoyferH2 forward (5'-GCT TTT ATC TCT CGC CCG TTG-3') and SoyferH2 reverse (5'-CAT TGT GTG CAA TCG GAA CAG C-3') primers were used. Transcript levels were normalized to the expression levels of alpha-Tubulin, as determined using the primers alpha-Tubulin forward ( $5^{\prime}$ TCT TCC ACC CTG AGC AGC TC-3') and alpha-Tubulin reverse ( $5^{\prime}$-AAC CTT GGA GAC CAG TGC AG-3'). The sizes of the amplified fragments were confirmed by agarose gel electrophoresis.

\section{METAL CONCENTRATION ANALYSIS}

A seed metal concentration analysis was performed according to the method of Masuda et al. (2008); Masuda et al. (2009). Brown seeds were collected randomly from the ear of the main tiller (the tiller in the center or the largest among all tillers in one plant). Ten seeds from each plant were dried overnight at $80^{\circ} \mathrm{C}$ in a heat drying machine. After determining the dry weight of each sample, the seeds were digested in $1 \mathrm{ml}$ of $13 \mathrm{M} \mathrm{HNO}_{3}$ and $1 \mathrm{ml}$ of $8.8 \mathrm{M} \mathrm{H}_{2}$
$\mathrm{O}_{2}$ (Wako, Osaka, Japan) at $200^{\circ} \mathrm{C}$ for 20 min with MARS Xpress (CEM, Matthews, NC, USA). After digestion, the samples were diluted to a volume of $5 \mathrm{ml}$ and analyzed via inductively coupled plasma atomic emission spectrometry (SPS1200VR; Seiko, Tokyo, Japan).

To obtain polished seeds, 30 brown seeds from the ear of the main tiller were placed into a 2-ml tube and shaken vigorously for $150 \mathrm{~s}$ at $2500 \mathrm{rpm}$ for four cycles using a Multi-Beads Shocker (Yasui Kikai, Osaka, Japan). Ten well polished seeds from each line were selected and dried overnight, weighed, digested, and then metal concentration was analyzed as described above.

Rice husks (approximately $100 \mathrm{mg}$ ) were also dried overnight, weighed, digested, and subjected to metal concentration analysis as described above.

\section{STATISTICS}

Student's $t$-test was used for each sample set to compare the data for the significant differences. The level of significance was set at $P<0.05$.

\section{RESULTS}

\section{PRODUCTION OF Fer-NAS-NAAT-IDS3 TRANSGENIC LINES}

To produce transgenic rice lines that concomitantly expressed soybean ferritin and barley enzymes for MAs biosynthesis, the transformation vector Fer-NAS-NAAT-IDS3 was produced (Figure 1A). This vector contained the OsGluB1 promoterSoyferH2, OsGlb promoter-SoyferH2, a 5-kb HvNAS1 genome fragment, an 11-kb $H v N A A T-A,-B$ genome fragment, and a 5$\mathrm{kb}$ IDS3 genome fragment in the marker-free vector $\mathrm{pBIMFN}$ (Nishizawa et al., 2006). This vector was used for rice

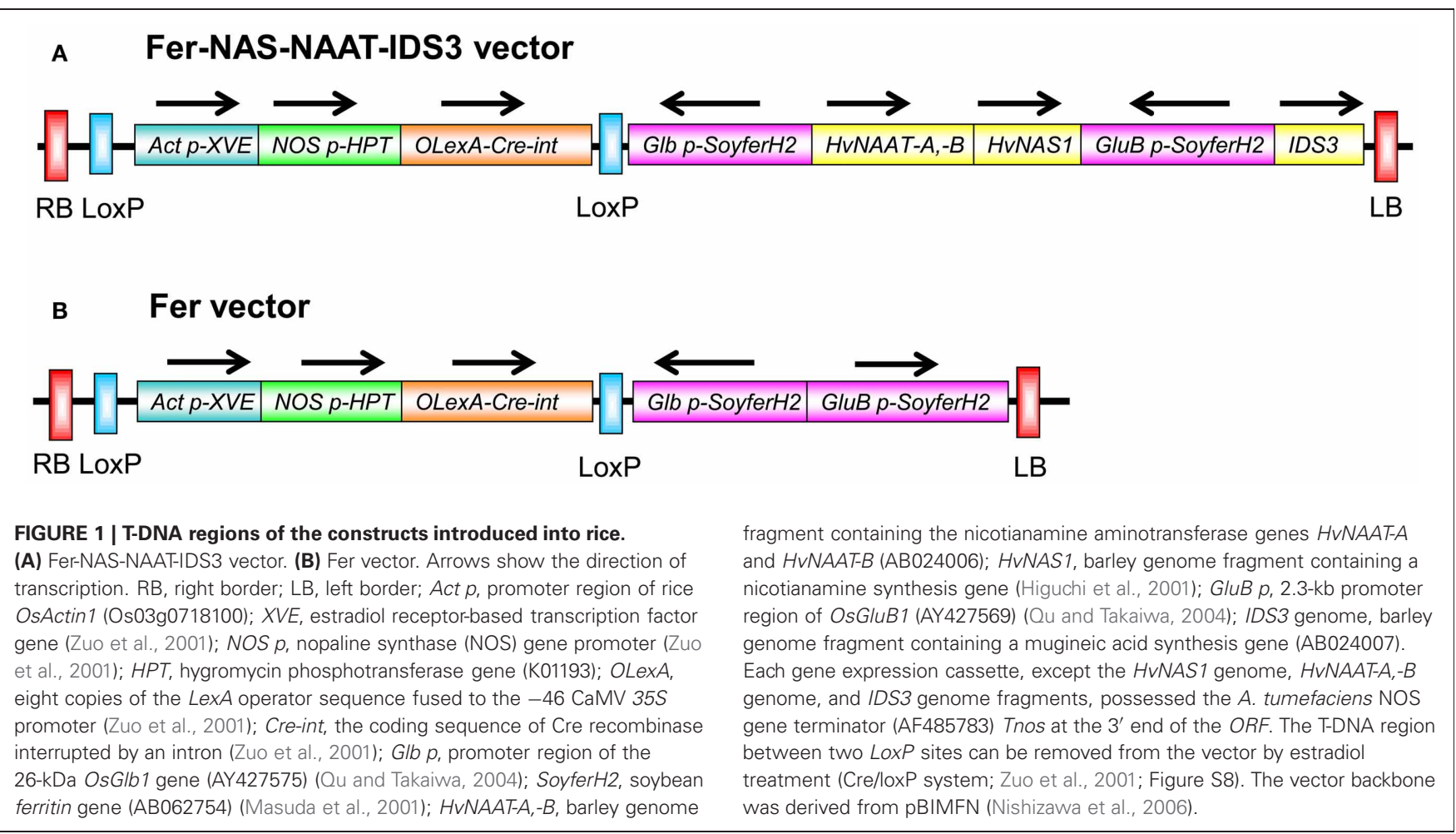




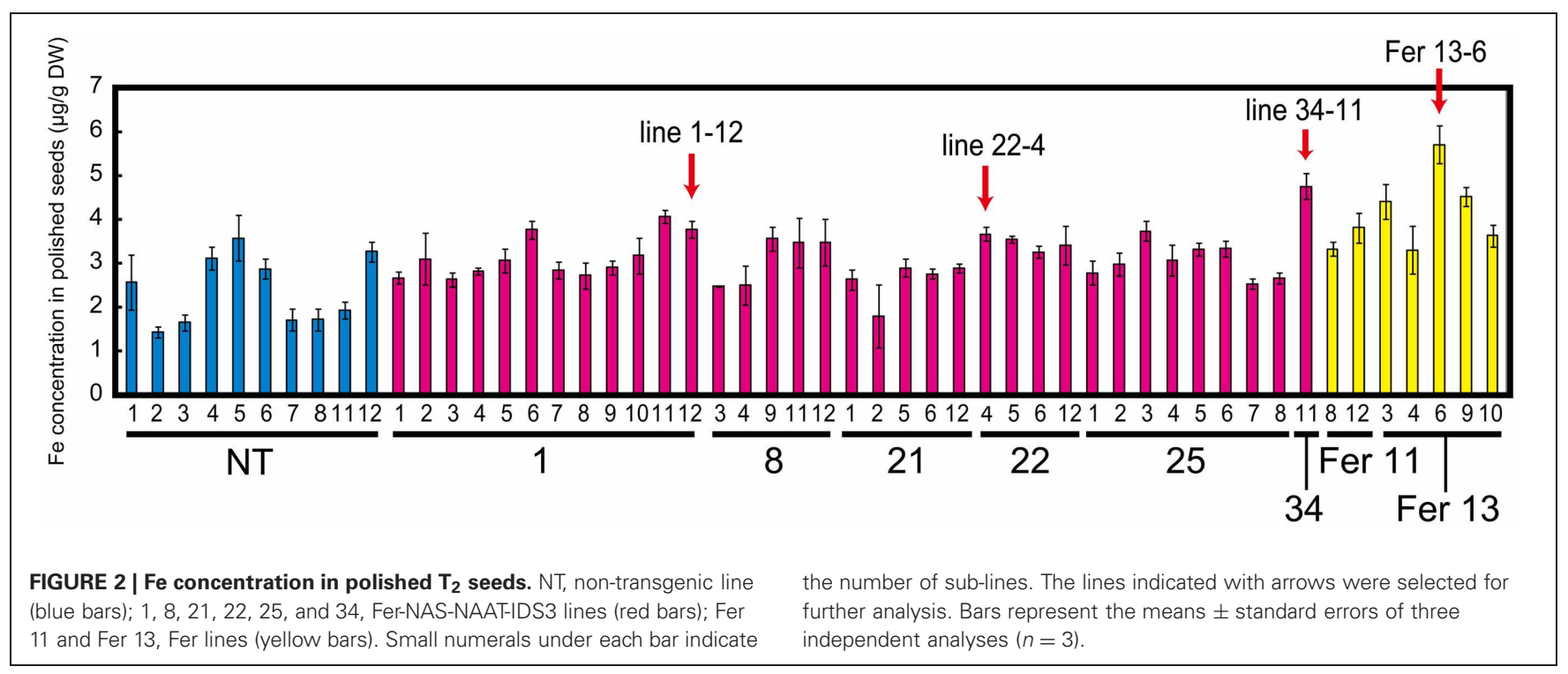

transformation and 102 lines were produced (referred to as FerNAS-NAAT-IDS3 lines).

To determine the advantage of introducing biosynthetic genes for MAs together with the ferritin gene, another rice transformation vector, Fer vector, which included the OsGluB1 promoterSoyferH2 and OsGlb promoter-SoyferH2 but no genes for MAs biosynthesis, was also produced (Figure 1B). Using this vector, 14 lines were obtained (referred to as Fer lines). The efficiency of transgenic plant production was lower in the Fer-NAS-NAATIDS3 lines than in the Fer lines and other transgenic lines of Tsukinohikari (data not shown). Among the transformants obtained, we screened desirable lines on the basis of the $\mathrm{Fe}$ concentration level in polished seeds rather than the transgene expression.

OsGluB1 promoter-SoyferH2 and IDS3 genome insertion in the Fer-NAS-NAAT-IDS3 lines was detected by genomic PCR. Among the 102 lines, insertion of the OsGluB1 promoterSoyferH 2 and IDS 3 was confirmed in Fer-NAS-NAAT-IDS3 lines $1,4,12,13,14,16,18,19,21,25,27,30$, and 34 (data not shown). Among the 14 Fer lines, insertion of the OsGluB1 promoterSoyferH2 was confirmed in lines 2, 11, 13, and 14 (data not shown). Next, the transgenic lines were cultivated in a greenhouse to obtain seeds.

\section{FE CONCENTRATION MEASUREMENT AND FERRITIN ACCUMULATION IN $\mathrm{T}_{\mathbf{1}}$ SEEDS}

Among the transformants obtained, we screened desirable lines on the basis of Fe concentration level in polished seeds rather than transgene expression. Therefore, after harvest, the Fe concentration in polished $\mathrm{T}_{1}$ seeds was measured. The Fe concentration was around two to three times higher in Fer-NAS-NAAT-IDS3 lines $8,14,21,22,25$, and 34 , as compared to that in the NT line (Figure S5). These Fer-NAS-NAAT-IDS3 lines and Fer lines 11 and 13 were cultivated in a greenhouse and the Fe concentration in polished $\mathrm{T}_{2}$ seeds was analyzed (Figure 2). Among the FerNAS-NAAT-IDS3 lines, 1-12, 22-4, and 34-11 showed especially

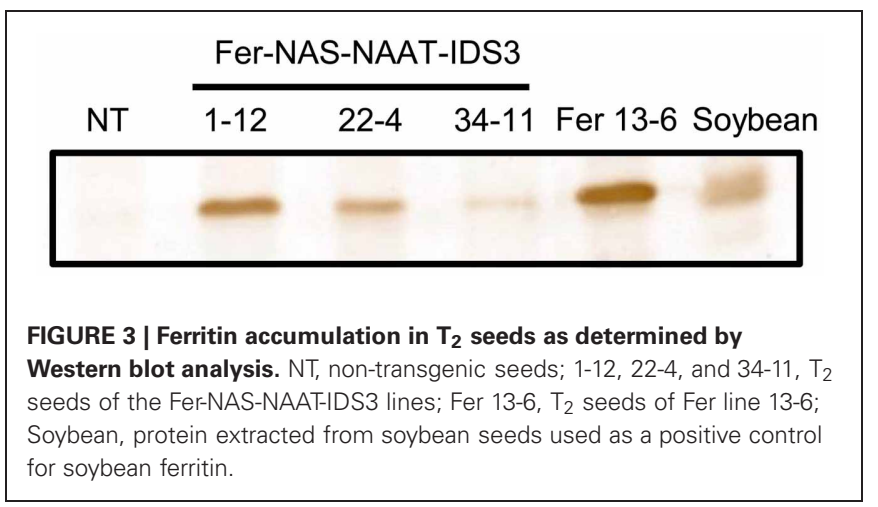

high Fe concentrations. These lines also exhibited normal yields (data not shown) and were therefore selected for further cultivation and detailed analysis. Among the Fer lines, 13-6 had a higher Fe concentration than the other Fer sub-lines. This line was also selected for further analysis. SoyferH2 accumulation in brown seeds of the selected lines (Fer-NAS-NAAT-IDS3 lines 1-12, 22-4, and 34-11, and Fer line 13-6) was confirmed (Figure 3).

HYDROPONIC CULTURE UNDER FE-DEFICIENT CONDITIONS AND GENE EXPRESSION PATTERNS OF HvNAS1, HvNAAT-A, HvNAAT-B, AND IDS3

To confirm the expression of $H v N A S 1, H v N A A T-A, H v N A A T-B$, and IDS 3 in the Fer-NAS-NAAT-IDS3 lines, plants were grown in hydroponic culture under both Fe-sufficient and -deficient conditions. After 1 week of Fe-deficient cultivation, the leaf color in Fer-NAS-NAAT-IDS3 lines 22-4 and 34-11 remained greener than that in the NT line (Figure 4A), as confirmed by the higher SPAD value (leaf chlorophyll index; Figure 4B). In contrast, the leaves of Fer line 13-6 were yellow and the SPAD value was lower than in the NT line and Fer-NAS-NAAT-IDS3 lines (Figures 4A,B).

After Fe-deficiency treatment, total RNA was extracted from the roots and shoots, and the expression of biosynthetic genes for 
A

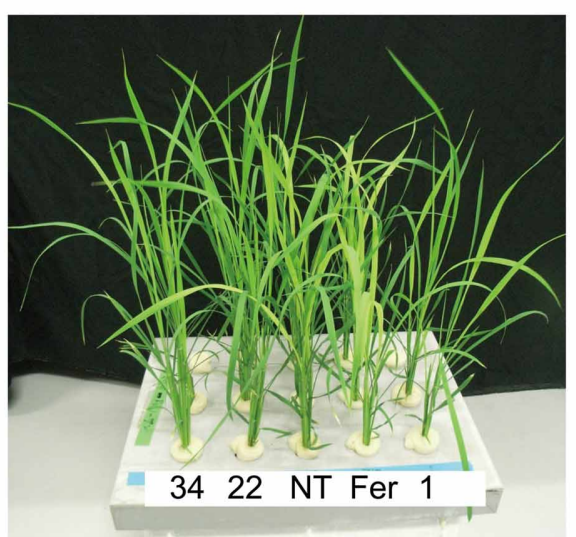

B

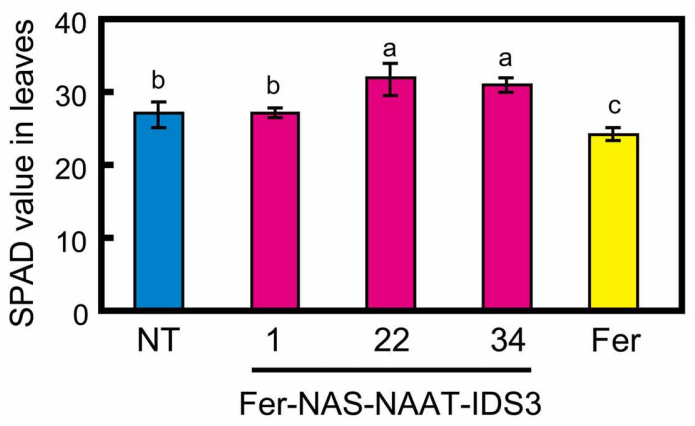

FIGURE 4 | Fe-deficiency symptoms in hydroponically grown transgenic lines. (A) Appearance of $T_{2}$ plants cultivated for 7 days in hydroponic culture solution without Fe. (B) The SPAD value of $T_{2}$ leaves under Fe-deficient conditions. NT, non-transgenic line; 1, 22, and 34, Fer-NAS-NAAT-IDS3 $T_{2}$ lines 1-12, 22-4, and 34-11,

respectively; Fer, Fer line 13-6. Bars represent the means \pm standard errors of six independent plants $(n=6)$. Different letters above the bars indicate significant differences $(P<0.05)$ by Student's $t$-test for each line.

MAs ( $H v N A S 1, H v N A A T-A, H v N A A T-B$, and IDS3) was analyzed by Northern blot analysis (Figure 5). In Fer-NAS-NAAT-IDS3 line 1-12, all of the introduced genes were expressed strongly in the roots, and they were also expressed in the shoots. In Fer-NASNAAT-IDS3 lines 22-4 and 34-11, IDS3 expression was detected as same as line 1-12. On the other hand, $H v N A A T-A$ and $-B$ expression was not detected in lines 22-4 and 34-11. In these lines, weak bands were detected by HvNAS1 hybridization, but the band strength was similar to that in the NT line and Fer line 13-6, in which HvNAS1 was not introduced. These weak bands might be derived from endogenous OsNAS1 expression, which could be hybridized to the HvNAS1 probe because of the strong similarity between $H v N A S 1$ and OsNAS1 ( $84 \%$ identity). To confirm the reason for the lack of strong $H v N A S 1, H v N A A T-A$, and $H v N A A T-B$ expression in lines 22-4 and 34-11, insertion of these genes was verified by genomic PCR (Figure S6). The insertion of all of these genes was detected in Fer-NAS-NAAT-IDS3 line 1 , but not in lines $22-4$ and $34-11$, or in the NT line and Fer line 13-6.

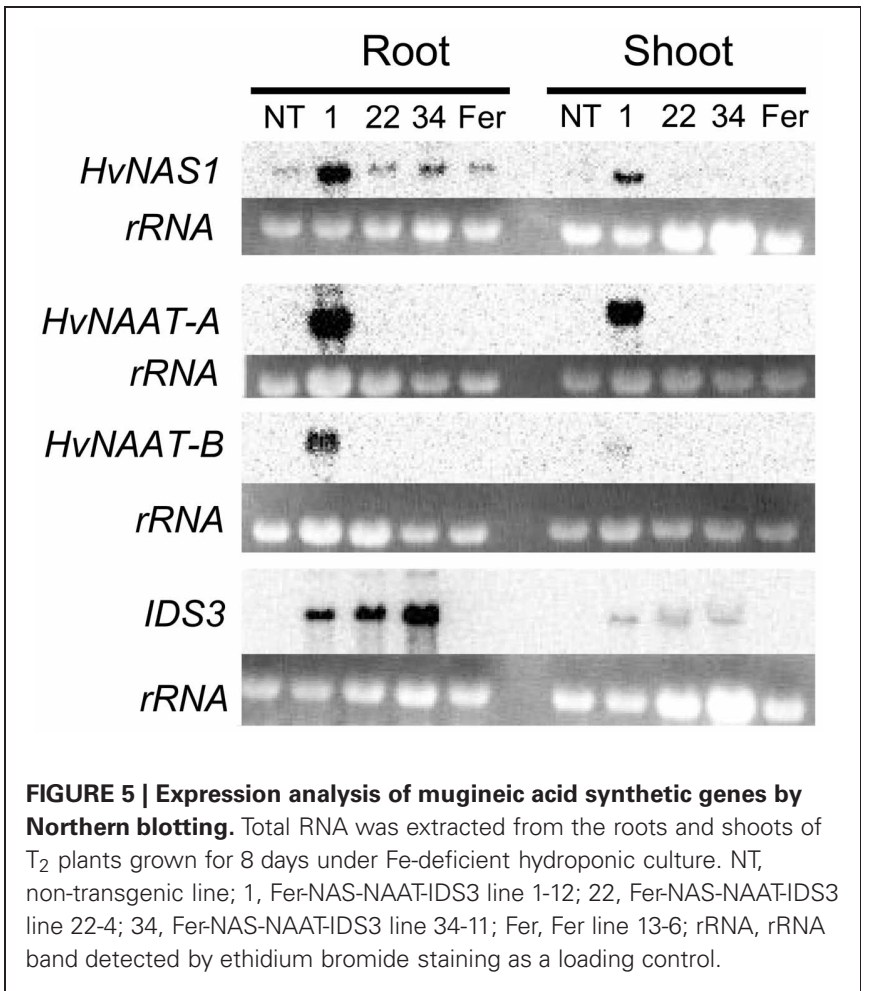

\section{GROWTH IN CALCAREOUS SOIL}

Suzuki et al. (2008) showed that rice lines with introduced barley HvNAS1 or the IDS3 genome fragment grew better than NT plants in calcareous soil. Therefore, we expected that FerNAS-NAAT-IDS3 rice would also exhibit Fe-deficiency tolerance, which might be distinguishable in calcareous soil. Therefore, NT plants and $\mathrm{T}_{2}$ plants of Fer-NAS-NAAT-IDS3 lines 1-12, 22-4, 3411 and Fer line 13-6 were grown in both commercially supplied soil (Fe-sufficient conditions) and calcareous soil (Fe-deficient conditions) in a greenhouse (Figure 6A). The shoot height was higher in Fer-NAS-NAAT-IDS3 lines 22-4 and 34-11 than in the NT line and Fer line 13-6 at 30 days after transplanting (DAT) in calcareous soil (Figure 6B). At around 60 DAT, the shoots of FerNAS-NAAT-IDS3 lines 22-4 and 34-11 were 15-20 cm higher than those of the NT line and Fer line 13-6. The SPAD values were also higher in Fer-NAS-NAAT-IDS3 lines 1-12, 22-4, and 34-11 than in the NT line and Fer line 13-6 at around 10-30 DAT. The SPAD value was lowest in Fer line 13-6 among all of the lines after 26 DAT (Figure 6C).

\section{FERRITIN ACCUMULATION AND FE CONCENTRATION IN T 3 SEEDS}

Ferritin accumulation was observed in $\mathrm{T}_{3}$ seeds of Fer-NASNAAT-IDS3 lines 1-12, 22-4, and 34-11, and in Fer line 13-6 seeds by Western blot analysis (Figure 7). Next, the metal concentrations in $\mathrm{T}_{3}$ seeds were analyzed (Figures 8, 9). After calcareous soil cultivation, the average Fe concentrations in Fer-NAS-NAATIDS3 lines 1-12, 22-4, and 34-11, and in Fer line 13-6 were 4.0, 4.0, 4.9 , and $3.3 \mu \mathrm{g} / \mathrm{g}$, respectively, which is higher than that in the NT line $(2.0 \mu \mathrm{g} / \mathrm{g})$ (Figure 8A). After commercially supplied soil cultivation, the average Fe concentrations in Fer-NAS-NAAT-IDS3 


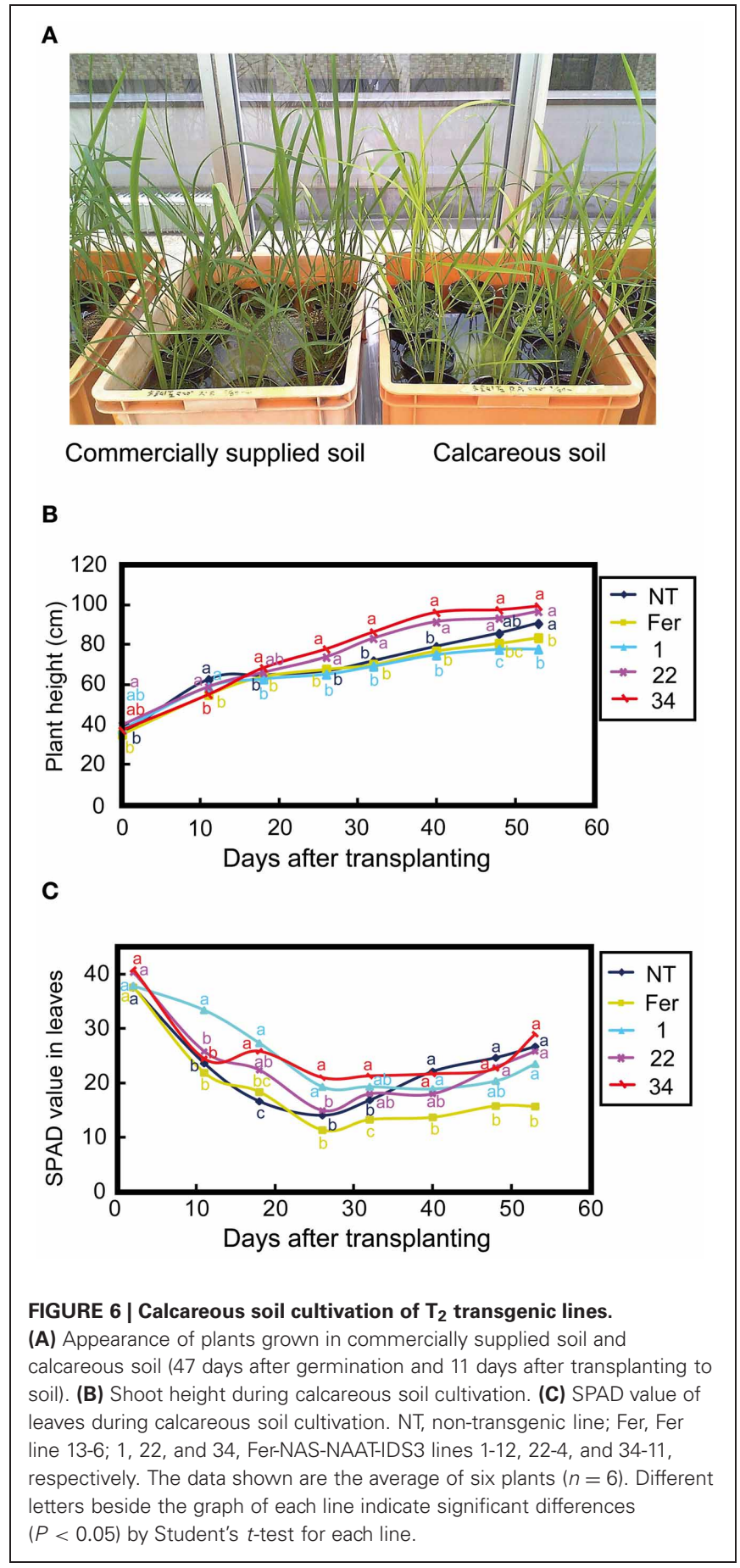

lines 1-12, 22-4, and 34-11, and in Fer line 13-6 were 2.5, 3.4, 4.0, and $3.3 \mu \mathrm{g} / \mathrm{g}$, respectively, which is also higher than that in the NT line $(1.1 \mu \mathrm{g} / \mathrm{g})$ (Figure 8B). The Fe concentrations in brown seeds of Fer-NAS-NAAT-IDS3 lines 1-12, 22-4, and 34-11 were also higher than that in the NT line following cultivation in either calcareous soil or commercially supplied soil (Figures 8C,D). There was no difference in the Fe concentration in brown seeds between the Fer and NT lines. The Zn concentrations in polished and brown seeds were up to 30\% higher in Fer-NAS-NAAT-IDS3 lines

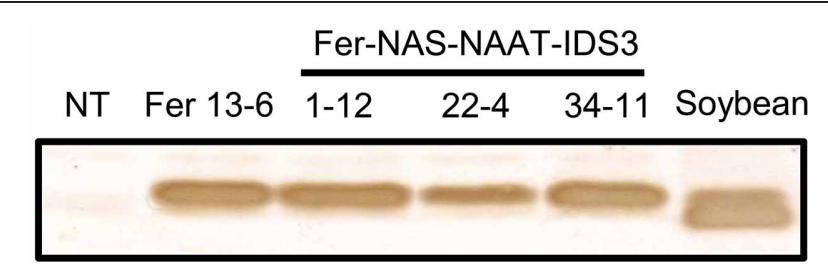

FIGURE 7 | Ferritin accumulation in $\mathbf{T}_{\mathbf{3}}$ seeds. Total protein was extracted from the seeds of six independent $T_{3}$ plants for each line cultivated in calcareous soil, and ferritin was detected by Western blot analysis. NT, non-transgenic seeds; $1-12,22-4$, and 34-11, $T_{3}$ seeds of the corresponding Fer-NAS-NAAT-IDS3 lines; Fer 13-6, T3 seeds of Fer line 13-6; Soybean, protein extracted from soybean seeds as a positive control for soybean ferritin.

1-12, 22-4, and 34-11, as compared to the NT and Fer lines following cultivation in calcareous soil or commercially supplied soil (Figures 9A-D). There was no remarkable difference in the husk Fe or Zn concentration between the transgenic and NT lines (Figures 8E,F and 9E,F).

In addition to $\mathrm{Fe}$ and $\mathrm{Zn}$ concentrations, the $\mathrm{Fe}$ and $\mathrm{Zn}$ contents in endosperm per seed were also higher in Fer-NASNAAT-IDS3 lines than in Fer or NT lines following cultivation in both calcareous and commercially supplied soil (Figure S7). Fe and $\mathrm{Zn}$ content in bran also tended to increase in Fer-NAS-NAATIDS3 lines, with line 34-11 showing significant increase compared to NT lines.

\section{DISCUSSION}

\section{GENERATION OF Fer-NAS-NAAT-IDS3 VECTOR}

To generate new Fe-fortified rice with improved growth under conditions of low Fe availability, we produced transgenic rice lines in which the soybean ferritin gene and barley genes responsible for MAs biosynthesis were introduced (Figure 1A). We designed the expression vector Fer-NAS-NAAT-IDS3 with the following issues in mind.

Soybean seeds possess two types of ferritin proteins: SoyferH1 $(26.5 \mathrm{kDa})$ and SoyferH2 (28 kDa) (Masuda et al., 2001). SoyferH1 can be digested by proteases, which may alter its structure, allowing Fe release, while SoyferH2 is more resistant to protease digestion than SoyferH1 (Masuda et al., 2001). Thus, we assumed that the stable ferritin, SoyferH2, would be more suitable for $\mathrm{Fe}$ accumulation in rice seeds. Therefore, we used SoyferH2 instead of SoyferH1 for expression in rice endosperm, which is similar to our previous report (Masuda et al., 2012). In fact, two ferritin bands appeared in soybean seed protein by Western blot analysis, among which the upper band $(28 \mathrm{kDa})$ matched the ferritin bands in our Fer-NAS-NAAT-IDS3 and Fer lines (Figures 3, 7).

Qu et al. (2005) reported that transgenic seeds with introduced ferritin under the control of both the 1.3-kb OsGluB1 promoter and $O s G l b$ promoter showed increased accumulation of ferritin compared to those with ferritin expressed under the control of either the 1.3-kb OsGluB1 promoter or the OsGlb promoter. In the present study, SoyferH2 was expressed under the control of both the 2.3-kb OsGluB1 promoter and the OsGlb 

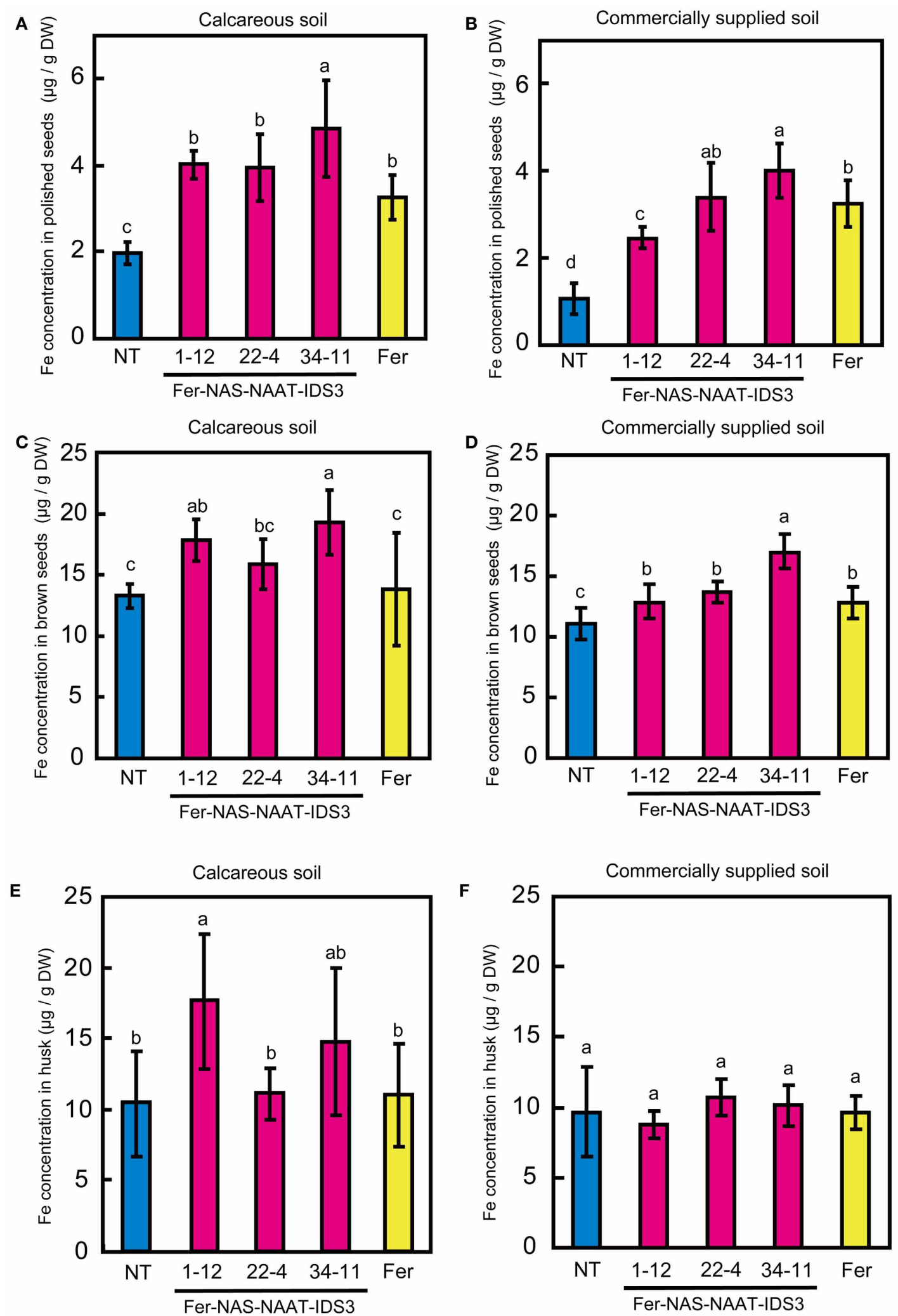

FIGURE 8 | Fe concentration in $T_{3}$ seeds. (A,B) Polished seeds. (C,D) Brown seeds. (E,F) Husk. Plants were cultivated in calcareous soil (A, C, and E) or commercially supplied soil (B, D, and F). Bars represent the means \pm standard errors of six independent

plants $(n=6)$. NT, non-transgenic line; 1-12, 22-4, and 34-11, Fer-NAS-NAAT-IDS3 $T_{3}$ lines; Fer, Fer $T_{3}$ line 13-6. Different letters above the bars indicate significant differences $(P<0.05)$ by Student's t-test for each line. 

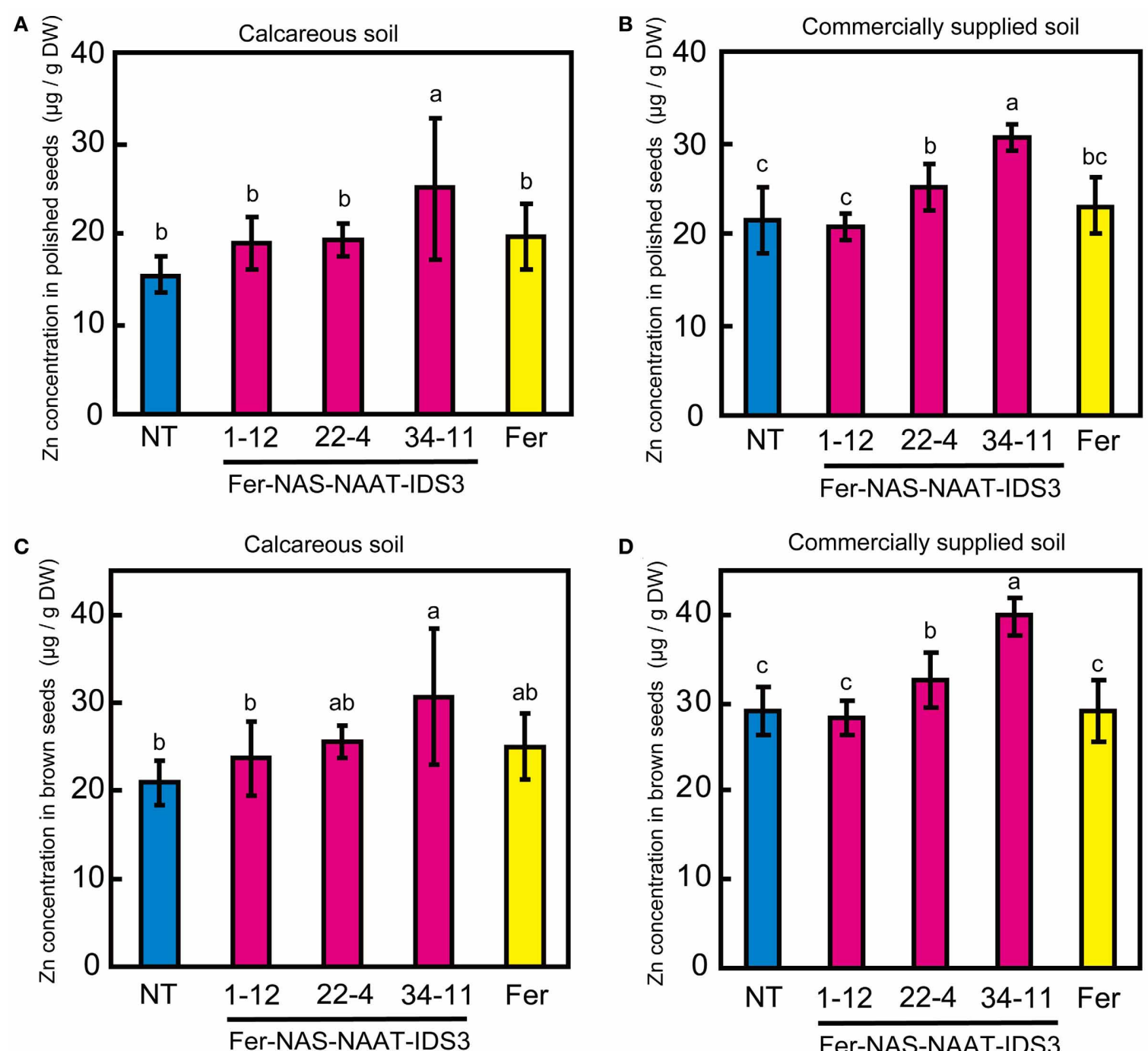

D
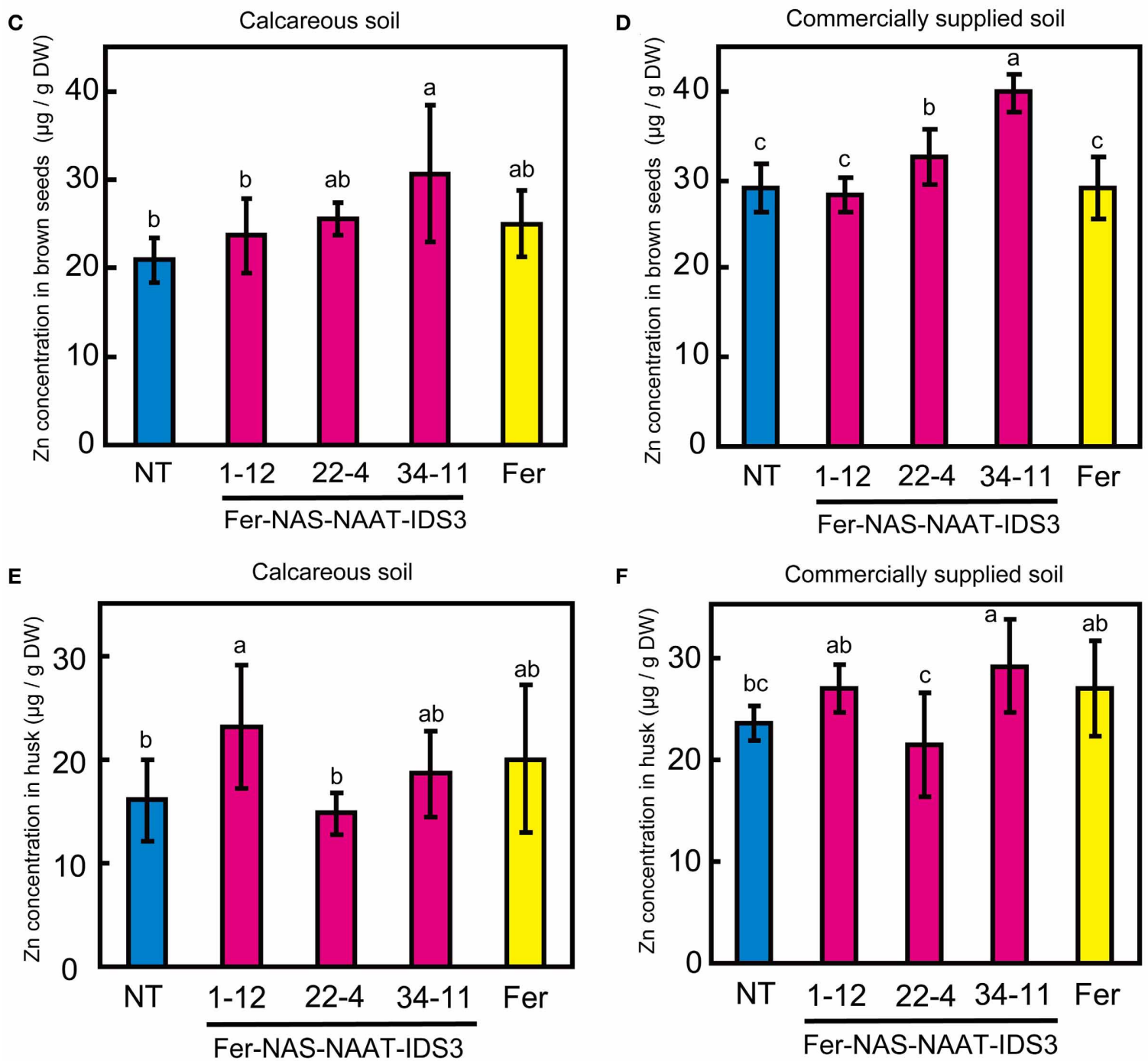

$\mathbf{F}$

Commercially supplied soil

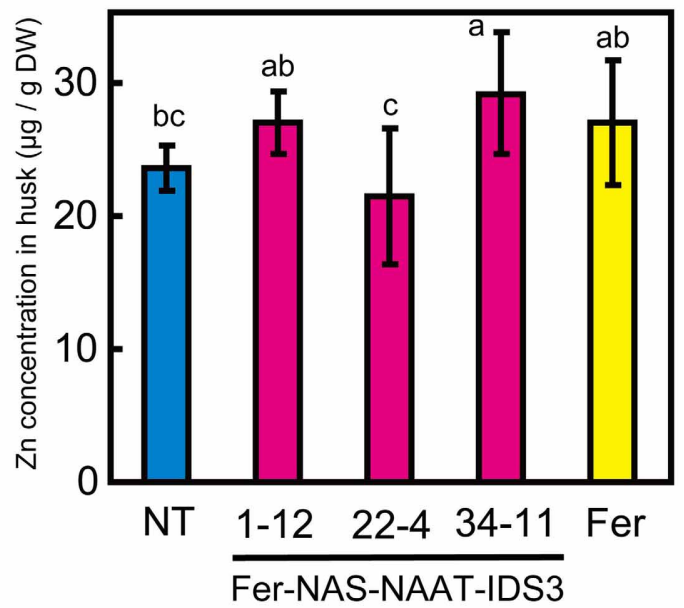

FIGURE $9 \mid \mathrm{Zn}$ concentration in $\mathbf{T}_{\mathbf{3}}$ seeds. (A,B) Polished seeds. (C,D) Brown seeds. (E,F) Husk. Plants were cultivated in calcareous soil (A, C, and E) or commercially supplied soil (B, D, and F). Bars represent the means \pm standard errors of six independent

plants $(n=6)$. NT, non-transgenic line; 1-12, 22-4, and 34-11, Fer-NAS-NAAT-IDS3 $\mathrm{T}_{3}$ lines; Fer, Fer $\mathrm{T}_{3}$ line 11-6. Different letters above the bars indicate significant differences $(P<0.05)$ by Student's t-test for each line. 
promoter (Figure 1) in order to achieve high ferritin accumulation in seeds, which is similar to our previous report (Masuda et al., 2012).

For the expression of genes for MAs biosynthesis in rice, the introduction of barley genomic fragments containing the corresponding genes and their promoters has been proven to be highly effective (Higuchi et al., 2001; Kobayashi et al., 2001). However, the introduction of multiple genomic fragments requires a large T-DNA insert, which causes difficulty in transformation. To solve this problem, pBIGRZ1 was used as a suitable binary vector because it allows the introduction of large insertions into the rice genome (Kawasaki, 2003). Using this vector as a backbone, pBIMFN (marker-free vector) was produced (Nishizawa et al., 2006). This vector, which utilizes the Cre/loxP system, allows unnecessary portions of the TDNA to be removed at any time from Fer-NAS-NAAT-IDS3 lines through estradiol treatment (Zuo et al., 2001) (Figure S8). Usuda et al. (2009) produced transgenic rice lines using this marker-free vector, and successfully removed the marker region from selected transgenic rice lines through estradiol treatment of the seeds. After removal of the two loxP regions from FerNAS-NAAT-IDS3 lines, all of the remaining transgenes would be derived from rice, soybean, and barley. This should make crops produced using this method easier for the public to accept, as compared to those created using the original binary vector, which includes bacterial selection marker genes such as that encoding hygromycin phosphotransferase or neomycin phosphotransferase II.

\section{CONFIRMATION OF INSERTED GENES}

Expression of all transgenes, including SoyferH2, HvNAS1, HvNAAT-A, HvNAAT-B, and IDS3, was observed only in line 112 (Figures 3, 5, and 7). Lines 22-4 and 34-11 did not possess the $H v N A S 1, H v N A A T-A$, and HvNAAT-B transgenes, although IDS3 expression and ferritin accumulation were observed (Figure S6). In the present study, the Fer-NAS-NAAT-IDS3 construct introduced into rice was large: about $35 \mathrm{~kb}$ between the right and left borders (Figure S8). Nakano et al. (2005) introduced a 92$\mathrm{kb}$ wheat genome fragment into rice by Agrobacterium-mediated transformation, but none of the four transgenic rice lines possessed the entire sequence; instead, fragments had been inserted. In addition to the $5^{\prime}$ or $3^{\prime}$ sides of the transgene that were missing, the central parts of the transgenes were also found to be missing. Similarly, the insertion of fragments is thought to have occurred in our Fer-NAS-NAAT-IDS3 transgenic lines. The IDS3 transcripts and ferritin proteins expressed in lines 1-12, 22-4, and 34-11 were similar in size, suggesting a lack of deletion in the expression cassettes for these transgenes (Figures 3, 5, and 7). Lines 22-4 and 34-11 may have lost the central part of the Fer-NAS-NAAT-IDS3 vector, between the HvNAS1 genome fragment and $H v N A A T-A,-B$ genome fragment. Nevertheless, these lines, as well as line 1-12, showed effective $\mathrm{Fe}$ accumulation in polished seeds both under Fe-sufficient and -deficient conditions (Figures 8A,B), along with improved tolerance to $\mathrm{Fe}$ deficiency (Figure 6), providing promising candidates for future applications as Fe-fortified crops that can tolerate Fe-limiting environments.

\section{THE SINGLE INTRODUCTION OF ferritin CAUSED SENSITIVITY TO FE DEFICIENCY BUT COULD BE OVERCOME BY THE CONCOMITANT INTRODUCTION OF BIOSYNTHETIC GENES FOR MAS}

Under both hydroponic culture and calcareous soil cultivation, Fer-NAS-NAAT-IDS3 lines 1-12, 22-4, and 34-11 showed Fe-deficiency tolerance (Figures 4, 6). Interestingly, Fer line 13 showed the opposite phenotype: sensitivity to Fe deficiency (Figures 4, 6). Wuytswinkel et al. (1999) reported that the overexpression of ferritin in tobacco caused abnormal Fe localization and symptoms of Fe deficiency. In our transgenic lines, SoyferH2 were expressed in Fe-deficient leaves of FC and FerNAS-NAAT-IDS3 lines (Figure S9). Data produced using a $44 \mathrm{~K}$ rice microarray also showed that OsGluB1 (Os02g0249900) and OsGlb (Os05g0499100) were expressed weakly in the leaves of plants grown under Fe-sufficient and -deficient conditions (data not shown). Thus, ectopic ferritin expression in the leaves of the Fer line under conditions of Fe deficiency might cause the accumulation and sequestration of $\mathrm{Fe}$, which is needed for growth, leading to an $\mathrm{Fe}$ deficiency-sensitive phenotype. This adverse effect was successfully complemented by the concomitant introduction of biosynthetic genes for MAs, as shown in the Fer-NASNAAT-IDS3 lines and also in previous reports. Lee et al. (2009b) reported that rice lines with enhanced OsNAS3 expression showed Fe-deficiency tolerance in addition to an increased Fe concentration in seeds. The overexpression of NAS genes together with ferritin may also help to avoid Fe deficiency sensitivity caused by introduction of the ferritin gene.

In the Fer-NAS-NAAT-IDS3 lines, introduction of the IDS3 genome fragment is thought to be responsible for the avoidance of sensitivity and further tolerance to Fe deficiency, because lines 224 and 34-11 expressed only IDS3 among the biosynthetic genes for MAs introduced. Suzuki et al. (2008) reported that introduction of the IDS3 genome fragment into rice conferred Fe-deficiency tolerance in field cultivation. This effect may be attributed to both enhanced production of MAs (DMA plus MA) and increased stability of Fe(III)-MA, as compared to Fe(III)-DMA under some conditions (von Wirén et al., 2000; Kobayashi et al., 2001).

\section{THE COMBINED INTRODUCTION OF ferritin AND IDS3 ENABLES EFFECTIVE FE AND ZN ACCUMULATION IN SEEDS}

In Fer-NAS-NAAT-IDS3 lines 22-4 and 34-11, even though the expression of $H v N A S 1, H v N A A T-A$, and $H v N A A T-B$ could not be detected, the Fe concentration in the seeds was the same or higher than that in line 1-12, which expressed all of the introduced genes for MAs biosynthesis ( HvNAS1, HvNAAT-A, HvNAAT-B, and IDS3) (Figures $\mathbf{5}$ and $\mathbf{8 A}, \mathbf{B}$ ). In previous field experiments, transgenic rice lines with introduced $H v N A S 1$ or $H v N A S 1$ plus $H v N A A T-A$ and $H v N A A T-B$ did not significantly increase the Fe concentration in seeds (Masuda et al., 2008; Suzuki et al., 2008). On the other hand, rice lines with the introduced IDS3 genome fragment showed an increased Fe concentration in polished seeds up to 1.25-1.4 times, as compared to that in the NT line in both Andosol soil, which has a normal pH, and calcareous soil (Masuda et al., 2008; Suzuki et al., 2008). Therefore, IDS3 is thought to be one of the most effective biosynthetic genes for MAs for the Fe biofortification of rice seeds. Rice possesses three HvNAS1 homologs (OsNAS1-3) and six HvNAAT-A and 
HvNAAT-B homologs (OsNAAT1-6) (Inoue et al., 2003, 2008). In contrast, rice lacks IDS3 homologs, which synthesize MA from DMA (Nakanishi et al., 2000; Kobayashi et al., 2001). The introduction of IDS3 to rice confers the ability to produce MA (Kobayashi et al., 2001), which could be advantageous for efficient Fe translocation within plants and might result in increased Fe accumulation in seeds, in addition to Fe-deficiency tolerance. Thus, the increased Fe concentration detected in Fer-NAS-NAATIDS3 lines 22-4 and 34-11, as compared to line 1-12, might have been caused by differences in the expression level or pattern of the IDS3 transgene (Figure 5).

The Fe concentration in brown seeds was increased by up to $30 \%$ in the Fer-NAS-NAAT-IDS3 lines but not in the Fer lines, as compared to the NT line under both calcareous soil and commercially supplied soil cultivation (Figures 8C,D). Because ferritin was expressed predominantly in the endosperm under the control of the OsGlb and $O s G l u B 1$ promoters, the contribution of ferritin expression to Fe accumulation is thought to be more obvious in polished seeds than in brown seeds. The concomitant introduction of IDS3 is thought to have contributed to Fe accumulation in brown seeds by enhancing Fe translocation.

The seed Fe concentration was higher in NT plants grown in calcareous soil, as compared to NT plants grown in commercially supplied soil (Figure 8). This may be because the yield in calcareous soil was low (data not shown) and Fe accumulated well in a limited number of seeds.

The seeds of the Fer-NAS-NAAT-IDS3 lines accumulated both $\mathrm{Fe}$ and $\mathrm{Zn}$. In contrast, Fer line 13-6 did not contain higher levels of $\mathrm{Zn}$ in its seeds, as compared to the NT line (Figure 9). Masuda et al. (2008) showed that the insertion of IDS3 into the rice genome increased the $\mathrm{Zn}$ concentration by $35 \%$ in polished seeds and by $29 \%$ in brown seeds in an Andosol field experiment. Suzuki et al. (2008) also showed that the Zn concentration was increased by $37 \%$ in brown seeds in a calcareous soil field experiment. Therefore, the increased Zn concentration in the FerNAS-NAAT-IDS3 lines may have been caused by the introduction of IDS3.

\section{REFERENCES}

Bashir, K., Inoue, H., Nagasaka, S., Takahashi, M., Nakanishi, H., Mori, S., et al. (2006). Cloning and characterization of deoxymugineic acid synthase genes from graminaceous plants. J. Biol. Chem. 281, 32395-32402.

Bashir, K., Ishimaru, Y., and Nishizawa, N. K. (2010). Iron uptake and loading into rice grains. Rice 3, 122-130.

Goto, F., Yoshihara, T., Shigemoto, N., Toki, S., and Takaiwa, F. (1999). Iron fortification of rice seed by the soybean ferritin gene. Nat. Biotechnol. 17, 282-286.

Gregorio, G. B., Senadhira, D., Htut, H., and Graham, R. D. (2000). Breeding for trace mineral density in rice. Food Nutr. Bull. 21, 382-386.

Grusak, M. A., and Cakmak, I. (2005). "Methods to improve the

Traditional breeding has also produced high Fe rice such as IR68144. Therefore, for further improvement of Fe concentration in seeds, it will be more efficient to generate higher Fe biofortified rice by transgenic method using a high Fe variety which has already been produced by traditional breeding.

In conclusion, transgenic rice expressing both ferritin and the barley MA synthase gene IDS3 showed increased Fe concentration when the plants were cultivated in both commercially supplied soil and calcareous soil. Fe-deficiency tolerance was also noted under calcareous soil cultivation. These results indicate that the concomitant introduction of ferritin and IDS3 is an effective way to biofortify seeds with Fe without causing Fe-deficiency symptoms under Fe-limited conditions. This method will be especially advantageous for use in Fe-limited environments, including those with a high soil $\mathrm{pH}$ and upland cultivation.

\section{ACKNOWLEDGMENTS}

We thank Dr. Mitsuru Abo (The University of Tokyo) for technical support for ICP-AES analysis, Dr. Kyoko Higuchi and Dr. Teppei Maruyama (Tokyo University of Agriculture) for assistance in rice cultivation. We also thank Ms. Reiko Nakanishi Itai and Dr. Motofumi Suzuki (The University of Tokyo) for technical advice regarding Northern blot analysis. We thank Dr. Fumiyuki Goto (CRIEPI, Japan) for providing soybean ferritin antibody, and Dr. Toshihiro Yoshihara (CRIEPI, Japan) and Dr. Fumio Takaiwa (NIAS, Japan) for providing OsGlb1 promoter, OsGluB1 promoter, and SoyferH2 gene. This work was supported by the Harvest Plus project, Core Research for Evolutional Science and Technology (CREST), and the Ministry of Education, Culture, Sports, Science and Technology (MEXT), Japan.

\section{SUPPLEMENTARY MATERIAL}

The Supplementary Material for this article can be found online at: http://www.frontiersin.org/Plant_Physiology/10.3389/ fpls.2013.00132/abstract

Hiei, Y., Ohta, S., Komari, T., and Kumashiro, T. (1994). Efficient transformation of rice (Oryza sativa L.) mediated by Agrobacterium and sequence analysis of the boundaries of the T-DNA. Plant J. 6, 271-282.

Higuchi, K., Suzuki, K., Nakanishi, H., Yamaguchi, H., Nishizawa, N. K., and Mori, S. (1999). Cloning of nicotianamine synthase genes, novel genes involved in the biosynthesis of phytosiderophores. Plant Physiol. 119, 471-479.

Higuchi, K., Watanabe, S., Takahashi, M., Kawasaki, S., Nakanishi, H., Nishizawa, N. K., et al. (2001). Nicotianamine synthase gene expression differs in barley and rice under Fe-deficient conditions. Plant J. 25, 159-167.

Inoue, H., Higuchi, K., Takahashi, M., Nakanishi, H., Mori, S., and
Nishizawa, N. K. (2003). Three rice nicotianamine synthase genes, OsNAS1, OsNAS2, and OsNAS3 are expressed in cells involved in longdistance transport of iron and differentially regulated by iron. Plant J. 36, 366-381.

Inoue, H., Kobayashi, T., Nozoye, T., Takahashi, M., Kakei, Y., Suzuki, K., et al. (2009). Rice OsYSL15 is an iron-regulated iron(III)deoxymugineic acid transporter expressed in the roots and is essential for iron uptake in early growth of the seedlings. J. Biol. Chem. 284, 3470-3479.

Inoue, H., Takahashi, M., Kobayashi, T., Suzuki, M., Nakanishi, H., Mori, S., et al. (2008). Identification and localization of the rice nicotianamine aminotransferase gene OsNAAT1 expression suggests the 
site of phytosiderophore synthesis in rice. Plant Mol. Biol. 66, 193-203.

Juliano, B. S. (1993). Rice in Human Nutrition. Los Baños: IRRI.

Kawasaki, S. (2003). New High Capacity Binary Shuttle Vector. Alexandria, VA: United States Patent Application Publication US 2003/0003585 Al.

Kobayashi, T., Nakanishi, H., Takahashi, M., Kawasaki, S., Nishizawa, N. K., and Mori, S. (2001). In vivo evidence that IDS3 from Hordeum vulgare encodes a dioxygenase that converts $2^{\prime}$ deoxymugineic acid to mugineic acid in transgenic rice. Planta 212, 864-871.

Lee, S., Chiecko, J. C., Kim, S. A., Walker, E. L., Lee, Y., Guerinot, M. L., et al. (2009a). Disruption of OsYSL15 leads to iron inefficiency in rice plants. Plant Physiol. 150, 786-800.

Lee, S., Jeon, U. S., Lee, S. J., Kim, Y. K., Persson, D. P., Husted, S., et al. (2009b). Iron fortification of rice seeds through activation of the nicotianamine synthase gene. Proc. Natl. Acad. Sci. U.S.A. 106, 22014-22019.

Lucca, P., Hurrell, R., and Potrykus, I. (2002). Fighting iron deficiency anemia with iron-rich rice. J. Am. Coll. Nutr. 21, 184-190.

Masuda, H., Ishimaru, Y., Aung, M. S., Kobayashi, T., Kakei, Y., Takahashi, M., et al. (2012). Iron biofortification in rice by the introduction of multiple genes involved in iron nutrition. Sci. Rep. 2:543. doi: 10.1038/srep00543

Masuda, H., Suzuki, M., Morikawa, K. C., Kobayashi, T., Nakanishi, H., Takahashi, M., et al. (2008). Increase in iron and zinc concentrations in rice grains via the introduction of barley genes involved in phytosiderophore synthesis. Rice 1 , 100-108.

Masuda, H., Usuda, K., Kobayashi, T., Ishimaru, Y., Kakei, Y., Takahashi, M., et al. (2009). Overexpression of the barley nicotianamine synthase gene $H v N A S 1$ increase iron and zinc concentrations in rice grains. Rice 2, 155-166.

Masuda, T., Goto, F., and Yoshihara, T. (2001). A novel plant ferritin subunit from soybean that is related to a mechanism in iron release. J. Biol. Chem. 276, 19575-19579.

Mayer, J. E., Pfeiffer, W. H., and Beyer, P. (2008). Biofortified crops to alleviate micronutrient malnutrition. Curr. Opin. Plant Biol. 11, 166-170.

Mihashi, S., and Mori, S. (1989). Characterization of mugineic acid$\mathrm{Fe}$ transporter in Fe-deficient barley roots using the multicompartment transport box method. Biol. Metals 2, 164-154.

Nakanishi, H., Yamaguchi, H., Sasakuma, T., Nishizawa, N. K., and Mori, S. (2000). Two dioxygenase genes, Ids 3 and $I d s 2$, from Hordeum vulgare are involved in the biosynthesis of mugineic acid family phytosiderophores. Plant Mol. Biol. 44, 199-207.

Nakano, A., Suzuki, G., Yamamoto, M., Turnbull, K., Rahman, S., and Mukai, Y. (2005). Rearrangements of large-insert T-DNAs in transgenic rice. Mol. Gen. Genomics 273, 123-129.

Nishizawa, N. K., Mori, S., and Nakanishi, H. (2006). Vector for Plant Transformation. Tokyo: Japan Patent, Patent Number 2006-191906

Ogo, Y., Itai, R. N., Nakanishi, H., Inoue, H., Kobayashi, T., Suzuki, M., et al. (2006). Isolation and characterization of IRO2, a novel ironregulated bHLH transcription factor in graminaceous plants. J. Exp. Bot. 57, 2867-2878.

Qu, L. Q., and Takaiwa, F. (2004). Evaluation of tissue specificity and expression strength of rice seed component gene promoters in transgenic rice. Plant Biotech. J. 2, 113-125.

Qu, L. Q., Yoshihara, T., Ooyama, A., Goto, F., and Takaiwa, F. (2005). Iron accumulation does not parallel the high expression level of ferritin in transgenic rice seeds. Planta 222, 225-233.

Stoltzfus, R. J., and Dreyfuss, M. L. (1998). Guidelines for the Use of Iron Supplements to Prevent and Treat Iron Deficiency Anemia. Washington DC: ILSI Press.

Suzuki, M., Morikawa, K. C., Nakanishi, H., Takahashi, M., Saigusa, M., Mori, S., et al. (2008). Transgenic rice lines that include barley genes have increased tolerance to low iron availability in a calcareous paddy soil. Soil Sci. Plant Nutr. 54, 77-85.

Suzuki, M., Takahashi, M., Tsukamoto, T., Watanabe, S., Matsuhashi, S., Yazaki, J., et al. (2006). Biosynthesis and secretion of mugineic acid family phytosiderophores in zincdeficient barley. Plant J. 48, 85-97.

Takagi, S. (1976). Naturally occurring iron-chelating compounds in oatand rice-root washings. Soil Sci. Plant Nutr. 22, 423-433.

Takahashi, M., Yamaguchi, H., Nakanishi, H., Shioiri, T., Nishizawa, N. K., and Mori, S. (1999). Cloning two genes for nicotianamine aminotransferase, a critical enzyme in iron acquisition (Strategy II) in graminaceous plants. Plant Physiol. 121, 947-956.

Tsukamoto, T., Nakanishi, H., Uchida, H., Watanabe, S., Matsuhashi, S., Mori, S., et al. (2009). $\mathrm{Fe}$ Translocation in Barley as Monitored by a PositronEmitting Tracer Imaging System (PETIS): Evidence for the Direct Translocation of Fe from Roots to Young Leaves via Phloem. Plant Cell Physiol. 50, 48-57.

Usuda, K., Wada, Y., Ishimaru, Y., Kobayashi, T., Takahashi, M., Nakanishi, H., et al. (2009). Genetically engineered rice containing larger amounts of nicotianamine to enhance the antihypertensive effect. Plant Biotech. J. 7, 87-95.

Vasconcelos, M., Datta, K., Oliva, N., Khalekuzzaman, M., Torrizo, L., Krishnan, S., et al. (2003). Enhanced iron and zinc accumulation in transgenic rice with the ferritin gene. Plant Sci. 164, 371-378.

von Wirén, N., Khodr, H., and Hider, R. C. (2000). Hydroxylated phytosiderophore species from rye and barley possess an enhanced chelating efficiency and affinity for iron(III). Plant Physiol. 124, $1149-1157$.

WHO. (2002). The World Health Report. Geneva: WHO.

Wuytswinkel, O. V., Vansuyt, G., Grignon, N., Fourcroy, P., and Briat, J. F. (1999). Iron homeostasis alteration in transgenic tobacco overexpressing ferritin. Plant J. 17, 93-97.

Zuo, J., Niu, Q. W., Møller, S. G., and Chua, N. H. (2001). Chemicalregulated, site-specific DNA excision in transgenic plants. Nat. Biotech. 19, 157-161.

Conflict of Interest Statement: The authors declare that the research was conducted in the absence of any commercial or financial relationships that could be construed as a potential conflict of interest.

Received: 15 February 2013; accepted: 20 April 2013; published online: 14 May 2013.

Citation: Masuda H, Kobayashi T, Ishimaru Y, Takahashi M, Aung MS, Nakanishi H, Mori S and Nishizawa NK (2013) Iron-biofortification in rice by the introduction of three barley genes participated in mugineic acid biosynthesis with soybean ferritin gene. Front. Plant Sci. 4:132. doi: 10.3389/fpls.2013.00132

This article was submitted to Frontiers in Plant Physiology, a specialty of Frontiers in Plant Science.

Copyright () 2013 Masuda, Kobayashi, Ishimaru, Takahashi, Aung, Nakanishi, Mori and Nishizawa. This is an openaccess article distributed under the terms of the Creative Commons Attribution License, which permits use, distribution and reproduction in other forums, provided the original authors and source are credited and subject to any copyright notices concerning any third-party graphics etc. 